\title{
BMJ Open Balance of benefits and harms of different blood pressure targets in people with multiple chronic conditions: a quantitative benefit- harm assessment
}

\author{
Hélène E Aschmann, ${ }^{\oplus 1}$ Cynthia M Boyd, ${ }^{2}$ Craig W Robbins, ${ }^{\oplus 3,4,5,6,7}$ \\ Richard A Mularski, ${ }^{8,9,10}$ Wiley V Chan, ${ }^{11}$ Orla C Sheehan, ${ }^{12}$ Renée F Wilson, ${ }^{13}$ \\ Wendy L Bennett, ${ }^{14}$ Elizabeth A Bayliss, ${ }^{15,16}$ Tsung Yu, ${ }^{17}$ Bruce Leff, ${ }^{2}$ \\ Karen Armacost, ${ }^{18}$ Carol Glover, ${ }^{18}$ Katie Maslow, ${ }^{18,19}$ Suzanne Mintz, ${ }^{18,20}$ \\ Milo A Puhan ${ }^{1}$
}

To cite: Aschmann HE, Boyd CM, Robbins CW, et al. Balance of benefits and harms of different blood pressure targets in people with multiple chronic conditions: a quantitative benefit-harm assessment. BMJ Open 2019;9:e028438. doi:10.1136/ bmjopen-2018-028438

- Prepublication history and additional material for this paper are available online. To view these files, please visit the journal online (http://dx.doi. org/10.1136/bmjopen-2018028438).

Received 07 December 2018 Revised 16 July 2019 Accepted 25 July 2019
Check for updates

(C) Author(s) (or their employer(s)) 2019. Re-use permitted under CC BY-NC. No commercial re-use. See rights and permissions. Published by BMJ.

For numbered affiliations see end of article.

Correspondence to Professor Milo A Puhan; miloalan.puhan@uzh.ch

\begin{abstract}
Objective Recent studies suggest that a systolic blood pressure (SBP) target of $120 \mathrm{~mm} \mathrm{Hg}$ is appropriate for people with hypertension, but this is debated particularly in people with multiple chronic conditions (MCC). We aimed to quantitatively determine whether benefits of a lower SBP target justify increased risks of harm in people with MCC, considering patient-valued outcomes and their relative importance.
\end{abstract}

Design Highly stratified quantitative benefit-harm assessment based on various input data identified as the most valid and applicable from a systematic review of evidence and based on weights from a patient preference survey.

Setting Outpatient care.

Participants Hypertensive patients, grouped by age, gender, prior history of stroke, chronic heart failure, chronic kidney disease and type 2 diabetes mellitus. Interventions SBP target of 120 versus $140 \mathrm{~mm} \mathrm{Hg}$ for patients without history of stroke.

Primary and secondary outcome measures Probability that the benefits of a SBP target of $120 \mathrm{~mm} \mathrm{Hg}$ outweigh the harms compared with $140 \mathrm{~mm} \mathrm{Hg}$ over 5 years (primary) with thresholds $>0.6$ (120 $\mathrm{mm} \mathrm{Hg}$ better), $<0.4$ ( $140 \mathrm{~mm} \mathrm{Hg}$ better) and 0.4 to 0.6 (unclear), number of prevented clinical events (secondary), calculated with the Gail/National Cancer Institute approach.

Results Considering individual patient preferences had a substantial impact on the benefit-harm balance. With average preferences, $120 \mathrm{~mm} \mathrm{Hg}$ was the better target compared with $140 \mathrm{~mm} \mathrm{Hg}$ for many subgroups of patients without prior stroke, especially in patients over 75 . For women below 65 with chronic kidney disease and without diabetes and prior stroke, $140 \mathrm{~mm} \mathrm{Hg}$ was better. The analyses did not include mild adverse effects, and apply only to patients who tolerate antihypertensive treatment. Conclusions For most patients, a lower SBP target was beneficial, but this depended also on individual preferences, implying individual decision-making is important. Our modelling allows for individualised

\section{Strengths and limitations of this study}

- This is the first comprehensive benefit-harm assessment of systolic blood pressure targets in people with multiple chronic conditions (MCC), considering patient-valued outcomes and their relative importance.

- The model is based on the most valid and applicable data from a systematic review of evidence, and on a patient preference survey to inform the relative importance of outcomes.

- Analyses were stratified by age, gender and prevalent comorbidities, and additionally by individual preferences in a sensitivity analysis, to clarify whether the benefit-harm balance of systolic blood pressure targets depends on individual preferences.

- Due to lacking evidence we could not include all patient-valued outcomes, in particular mild adverse events - therefore, the results apply only to people who tolerate antihypertensive treatment.

- Techniques to measure the blood pressure differed between trials and may differ between trials and clinical practice, therefore, an interpretation of what would be the analogous targets in clinical practice may be required.

treatment targets based on patient preferences, age, gender and co-morbidities.

\section{BACKGROUND}

Recommended blood pressure targets for people with multiple chronic conditions (MCC) and hypertension vary across guidelines. ${ }^{1-4}$ The recent publication of three randomised clinical trials (RCTs), ACCORD in people with diabetes, ${ }^{5}$ SPRINT in people without diabetes ${ }^{6}$ and SPS3 in people with 
lacunar stroke, ${ }^{7}$ caused debate in the medical community about the benefits and harms of systolic blood pressure (SBP) targets lower than $140 \mathrm{~mm} \mathrm{Hg}$, since lower targets reduced the risk of some cardiovascular outcomes, but increased the incidence of adverse events, and because the effect on mortality differed across the RCTs. ${ }^{5-8}$ Concerns were voiced that the absolute benefit of lower SBP targets is small and may not outweigh the absolute harm, especially in older adults with MCC. ${ }^{9}$ Furthermore, techniques to measure the blood pressure differed between trials and may differ between trials and clinical practice. ${ }^{110}$

The question of which blood pressure target is optimal for people with MCC and whether this differs for some groups of people was recently identified as a priority question by people with MCC and caregivers in a multi-step, mixed-methods process involving guideline developers. ${ }^{11}$ The benefit-harm balance depends on the baseline incidence for different outcomes, the relative effect of different targets and the relative importance of potential benefit and harm outcomes to the patient. ${ }^{12}{ }^{13}$ Clinical guidelines often consider these three key elements when developing recommendations but often do so unsystematically and qualitatively. The National Cancer Institute has developed methods for quantitative benefit-harm assessment to elucidate the benefit-harm balance of treatment alternatives in a systematic and transparent way. ${ }^{13} 14$ Using such methods, the absolute beneficial and harmful effects on different outcomes are weighed and compared on a common scale.

In order to determine the benefit-harm balance of different blood pressure targets specifically for people with MCC, and to clarify whether the balance depends on baseline characteristics or outcome preferences, we performed quantitative assessments for a priori defined subgroups based on age, gender, prior history of stroke, chronic kidney disease (CKD), diabetes and chronic heart failure.

\section{METHODS}

\section{Study design}

We performed a quantitative benefit-harm assessment and followed the Consolidated Health Economic Evaluation Reporting Standards for cost-effectiveness analyses. ${ }^{15}$ We modelled the benefit-harm balance in different subgroups according to the Gail/National Cancer Institute approach ${ }^{13}$ that has been used before to assess various prevention treatments. ${ }^{16-19}$ This method calculates absolute risk differences of treatments (ie, the difference in expected number of events for each outcome) from baseline incidence and relative effects and weighs the absolute risk differences on different outcomes according to their importance relative to each other. Importantly, it considers competing risks (eg, death), and the statistical uncertainty of input parameters.

Definition of the target population, subgroups and outcomes The target population was people age 50 and older with hypertension and multiple chronic conditions in the
USA. Based on patient and caregiver input and clinical judgement, we predefined subgroups according to prevalence and possible impact on the benefit-harm balance: age, gender, prior history of ischaemic stroke, CKD, type 2 diabetes mellitus and chronic heart failure. Based on a literature review of outcomes that have been used in relevant $\mathrm{RCTs}^{5-7}$ augmented by qualitative research with patients and caregivers, ${ }^{11}$ we aimed to include the following patient-valued outcomes: acute kidney injury (AKI), CKD, end-stage renal disease (ESRD), stroke, death, treatment burden, cognitive impairment, injurious falls, hypotension, dizziness, syncope, myocardial infarction and heart failure.

\section{Evidence selection}

Relative effects of blood pressure targets on all outcomes Systematic search for RCTs on blood pressure targets

We systematically searched for RCTs on blood pressure targets. Since a number of systematic reviews were published recently we did not duplicate their entire search but identified relevant and recent systematic reviews first (figure 1). The detailed search methods are described in online supplementary figures S1 and S2, table S1. From the systematic reviews and update searches, we identified 20 RCTs of blood pressure targets in an adult population in a non-intensive care setting. We did not use any of the meta-analyses of the eight systematic reviews because they were either based on or standardised by achieved blood pressure, or included trials that did not compare blood pressure targets (but eg, one vs two antihypertensives), or focused on prehypertension.

\section{Inclusion and exclusion of RCTs on blood pressure targets}

We excluded three RCTs with high risk of bias, ${ }^{20-22}$ as assessed with the Cochrane Risk of Bias Tool $^{23}$ (see online supplementary table S2), 12 RCTs that did not report enough outcomes or events to judge the benefit-harm balance (eight reported no harm outcomes, ${ }^{24-31}$ two reported only CKD as a harm outcome ${ }^{32} 33$ and one reported only sparse data on benefit outcomes, ${ }^{34}$ and one reported only sparse data on harm outcomes ${ }^{35}$ ), one RCT because the study population was not our target population, ${ }^{36}$ and one because the targets that were compared were so high $(<150 / 85 \mathrm{vs}<180 / 105 \mathrm{~mm} \mathrm{Hg})$ that they were no longer clinically relevant (figure 1 and online supplementary figure S3) ${ }^{37}$ We included three RCTs: SPRINT, ${ }^{6}$ ACCORD ${ }^{5}$ which both compared $120 \mathrm{~mm} \mathrm{Hg}$ versus $140 \mathrm{~mm} \mathrm{Hg}$, and SPS3, ${ }^{78}$ which compared $<130 \mathrm{~mm} \mathrm{Hg}$ to 131 to $149 \mathrm{~mm} \mathrm{Hg}$. SPRINT included people without prior stroke and without diabetes, ACCORD included people with diabetes but without prior stroke and SPS3 included people with lacunar stroke. Notably, we could not include dizziness, cognitive impairment and treatment burden in our analysis due to lacking evidence. For treatment burden, we considered evidence on increased number of pills per day, more doctor appointments or burden due to diet and exercise. 


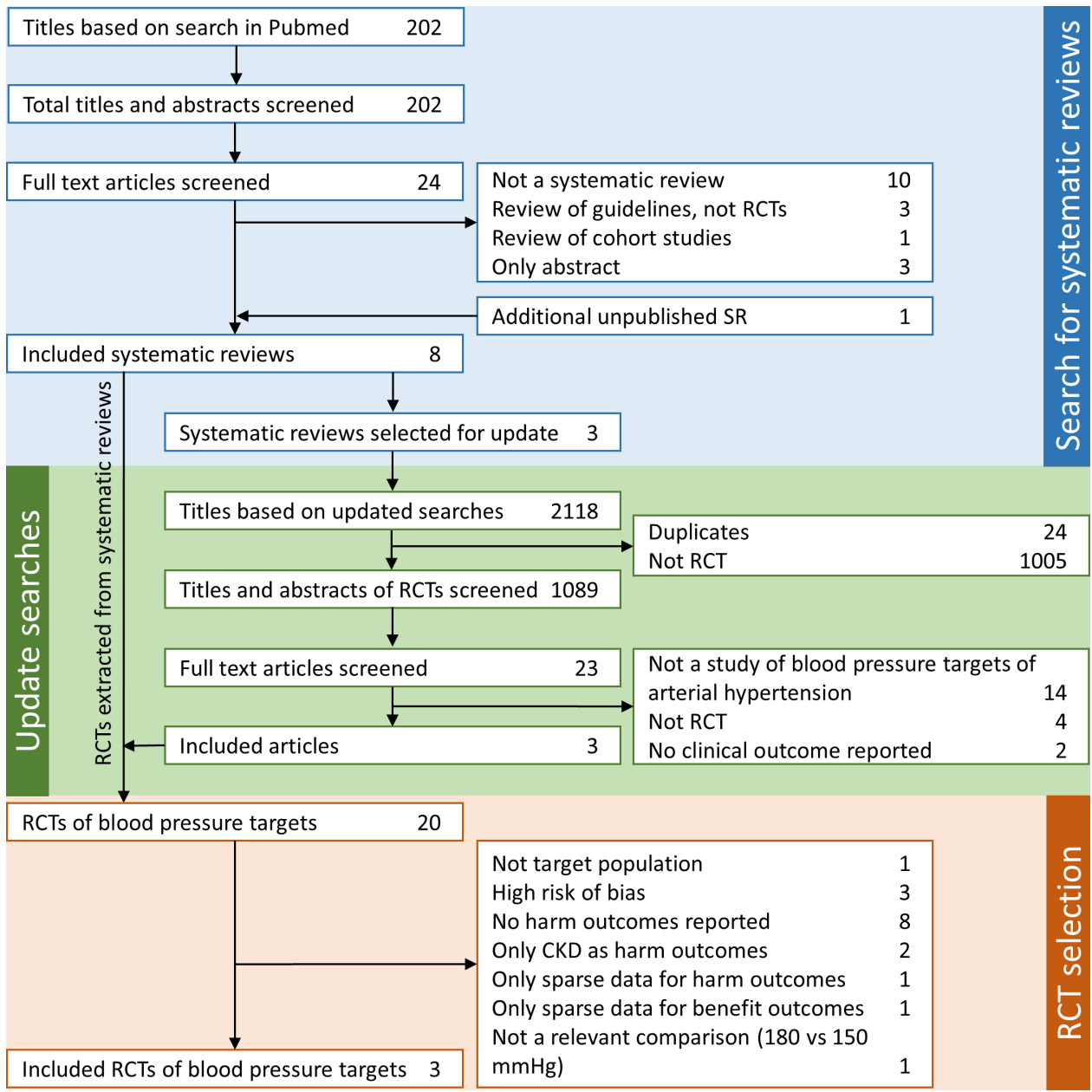

Figure 1 Flow chart of the search for RCTs comparing blood pressure targets based on existing systematic reviews and the RCT selection. CKD, chronic kidney disease; RCTs, randomised clinical trials; SR, systematic review.

\section{Considerations on effect modification and heterogeneity}

Our search indicated no relevant heterogeneity between subgroups in the publications of the trials, however, the relative risks reported by the three RCTs sometimes differed. In a few cases, this heterogeneity was clearly due to sparse data bias, and thus we corrected these estimates (table 1). ${ }^{38} 39$ The remaining heterogeneity was either due to different study populations or different outcome definitions. As a first step to ensure applicability, we did not pool the estimates from SPRINT, ACCORD and SPS3, but we applied the estimates to subgroups in our benefit-harm assessment according to the study population (ie, SPRINT to people without prior history of stroke and diabetes, ACCORD to people with diabetes and without stroke and SPS3 to people with stroke, respectively). In a second step, we made exceptions for a few outcomes, where we applied the estimates from SPRINT instead of ACCORD or vice-versa, when differences in estimates were judged to be due to the outcome definition rather than because of different study populations (table 1).

Because we decided to apply only estimates from SPS3 to people with prior history of stroke, and because important outcomes for patients with CKD were not reported in SPS3, namely AKI and ESRD, we did not calculate a benefit-harm balance for people with both a history of stroke and CKD, as there would be significant uncertainty in the balance.

\section{Baseline incidence of all outcomes}

To address baseline incidence of outcomes for people with hypertension according to age, gender and prior patient history (stroke, diabetes, heart failure and CKD), adapting a previously described method, ${ }^{12}$ we prioritised different sources in the following order:

1. US national registries that reflect the target population as closely as possible.

2. Observational studies with similar study population as the target population.

3. RCTs.

The latter were favoured the least because, compared with observational studies, restrictive inclusion criteria or recruitment mechanisms can substantially affect baseline incidence rates. To avoid inconsistency, we based as many estimates as possible on prediction models and incidence tables based on the Atherosclerosis Risk in Communities (ARIC) study. ${ }^{40-44}$ Assumptions about additional risk factors beyond those defining the subgroups were necessary when prediction models included other risk factors 
Table 1 Incidence rate ratios for the comparison of different systolic blood pressure targets

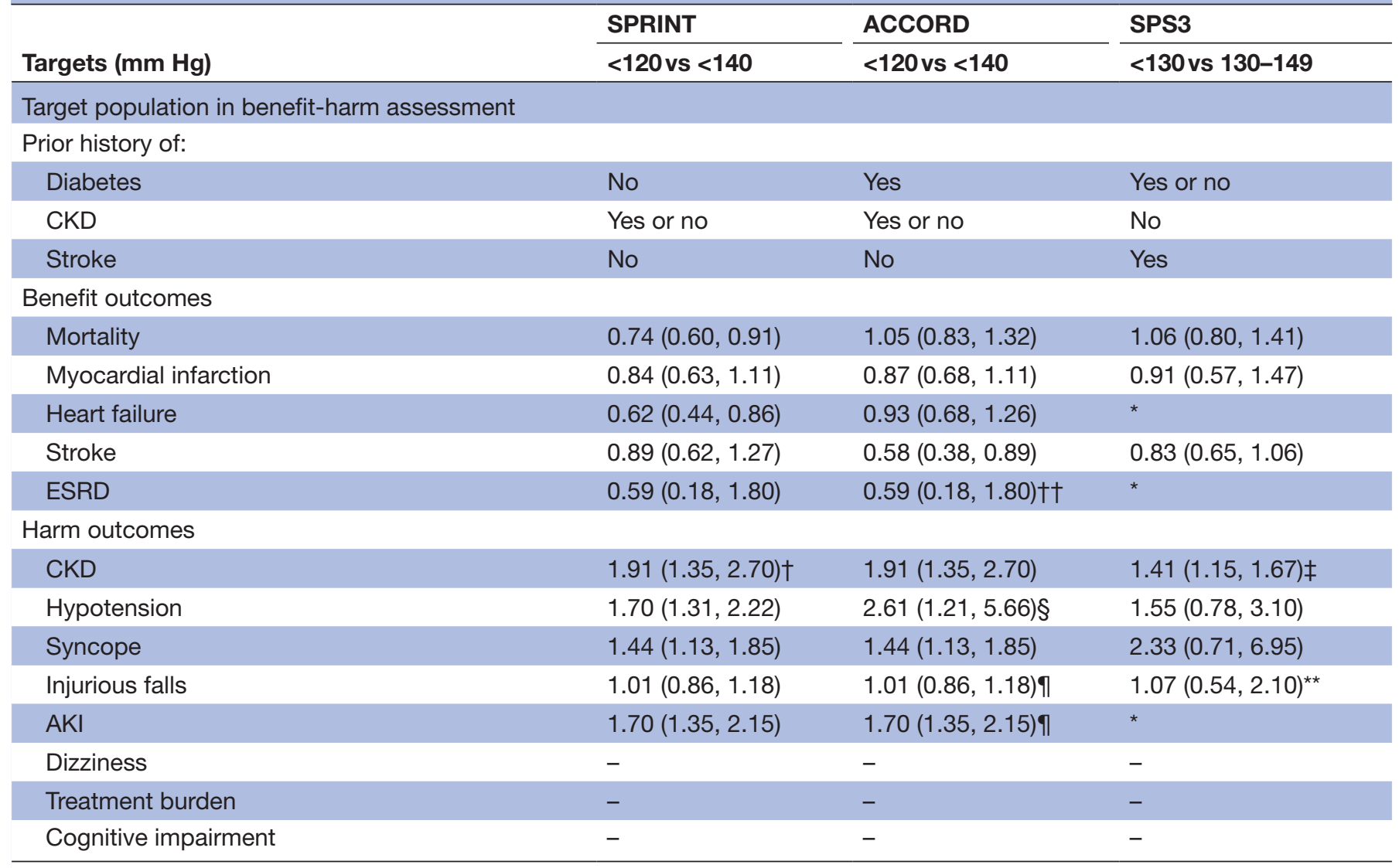

Incidence rate ratios calculated based on number of events and number of patient-years in each trial arm. Outcomes are grouped into benefit outcomes and harm outcomes according to the SPRINT estimate. Estimates larger than one imply that the outcome occurs more frequently with the lower blood pressure target. Conversely, estimates lower than one mean the lower blood pressure target reduces the risk for that outcome.

*SPS3 did not report heart failure, ESRD and AKI, and we did not extrapolate from SPRINT or ACCORD because the SBP targets were different.

†We used the CKD estimate from ACCORD instead of SPRINT, because an incident estimated glomerular filtration rate $<30 \mathrm{~mL} / \mathrm{min}^{2} 1.73 \mathrm{~m}{ }^{2}$ is more likely symptomatic and patient-important than $<60 \mathrm{~mL} / \mathrm{min} / 1.73 \mathrm{~m}^{2}$.

$\ddagger$ The estimate for CKD in SPS3 is an OR, extracted from a posthoc analysis of the trial data.

$\S$ Corrected for sparse data bias with a prior incidence rate ratio between 1 and 5 .

IWe used estimates from SPRINT for injurious falls and AKI because ACCORD did not report them, and we used the estimate from SPRINT for syncope because we assessed the ACCORD estimate as high risk of bias.

${ }^{* *}$ Corrected for sparse data bias with a prior incidence rate ratio between 0.5 and 2 . We could not include dizziness, treatment burden and cognitive impairment in our main analysis.

††We used the ESRD estimate from SPRINT instead of ACCORD because SPRINT considered only incident ESRD in patients who had prior history of CKD.

AKI, acute kidney injury; CKD, chronic kidney disease; ESRD, end-stage renal disease; OR, odds ratio; SBP, systolic blood pressure.

(see online supplementary table S3 for an overview on which subgroups affected the baseline incidence of which outcomes). We also developed a web-calculator in which the impact of all risk factors can be explored.

Relative importance of outcomes from patient preference survey We surveyed people with MCC and hypertension in the USA using best-worst scaling (Aschmann et al, 'Outcome preferences of older people with multiple chronic conditions and hypertension: A cross-sectional survey using best-worst scaling', submitted), a preference elicitation method which provides weights for outcomes relative to one another and is easy to understand..$^{45}$ Both the Institutional Review Boards of Johns Hopkins University and
Kaiser Permanente Colorado approved the survey. In brief, we sent paper surveys to members of Kaiser Permanente Colorado aged 60 or older who had hypertension and at least two other chronic conditions. We presented respondents with 11 blocks of five outcomes and asked them which they would worry about most, and which least. We analysed 207 responses with a conditional logit model. Based on this, we used the following weights in the main analysis: death (1), myocardial infarction (0.66), heart failure (0.65), stroke (0.76), ESRD (0.47), CKD $(0.45)$, hypotension $(0.01)$, syncope $(0.11)$, injurious falls (0.02), AKI (0.33), treatment burden (0). Respondents were aged 60 to 97 , and the most common conditions 
additionally to hypertension were hyperlipidaemia, CKD and diabetes. They were similar to non-respondents in terms of age, number of comorbidities, gender, race and ethnicity. Because baseline characteristics were not associated with different preferences, we applied the weights to all subgroups in the benefit-harm assessment, including people younger than 60 years.

\section{Statistical analysis}

We calculated the number of expected events over 5 years in a population of 10000 based on the baseline incidence and relative effects. ${ }^{13}$ Longer time horizons may be more relevant, but the RCTs were too short to inform relative risks and incidence rates for longer than 5 years, especially for adverse events like AKI or CKD. All-cause mortality was considered a competing risk for all other outcomes. We considered statistical uncertainty of estimates in relative effects and distributions in baseline incidences (due to distributions of risk factors accounted for in prediction models) in 100000 repetitions in the analysis (equations in online supplementary file).

Subsequently, we calculated the Gail index ${ }^{13}$ as the weighted difference in event numbers: the difference in number of expected events for each outcome is multiplied with its relative importance, and the weighted differences are summed across outcomes. For example, if we only considered stroke and CKD, and if we expect 58 less strokes but 201 more CKD events with the lower target, the Gail index would be $(-58) \times 0.76+201 \times 0.45=+46$, that is, there would be 46 more events of 'importance 1 ' with the lower target, thus the higher target would be better. However, to account for statistical uncertainty, we repeated the calculation of the Gail index 100000 times. In the example above, this would yield a distribution with a mean Gail index of +46 .

We then calculated the probability that one SBP target is better than another. In the example, the probability that the higher target is better is the proportion of the repetitions when the Gail index was greater than zero. For example, a probability of 0.7 that the higher target is better inversely means the probability is 0.3 that the lower target is better. We categorised the probabilities into greater than 0.6 (target better than reference), smaller than 0.4 (target worse than reference) and in between (unclear which is better).

To define a range of possible estimates of the benefit-harm balance, we performed two additional analyses - one favouring the lower and one the upper target taking into account (non-statistical) uncertainty in the baseline incidence.

\section{Sensitivity analyses}

To investigate the effect of individual preferences, we generated preference values for each respondent in the survey based on individual best-minus-worst scores (a count measure). In sensitivity analysis 1 , we applied the individual preference values. In sensitivity analysis 2 , we additionally included treatment burden, assuming $20 \%$ of people have more treatment burden with the lower target than with $140 \mathrm{~mm} \mathrm{Hg}$. In sensitivity analyses 3 to 5 we assigned different population preference weights (preferences on an aggregate level, see online supplementary table S4).

\section{Software}

We performed calculations in R V.3.3.1.

\section{Patient and public involvement}

The research question was identified as a priority question by people with MCC and caregivers. ${ }^{11}$ A preference survey among people with MCC informed on applicable weights for outcomes in the benefit-harm assessment. Four patient and caregiver co-investigators were involved throughout the project, starting with the conception of the study. In particular, they helped in the development of the survey, and contributed to framing the conclusions and to drafting a summary of the results that was sent to all survey respondents. They continue to help with the dissemination of the study results.

\section{RESULTS}

Table 2 shows the expected number of events over 5 years per 10000 people, across three age groups in people without prior history of stroke (mixed population with and without diabetes, CKD and heart failure) with SBP targets of $140 \mathrm{~mm} \mathrm{Hg}$ and $120 \mathrm{~mm} \mathrm{Hg}$. In 50 to 64 year olds 361 deaths are expected per 10000 people over 5 years with a target of $140 \mathrm{~mm} \mathrm{Hg}$, and 281 deaths are expected with a target of $120 \mathrm{~mm} \mathrm{Hg}$. Hence, 80 deaths per 10000 people 50 to 64 years old over 5 years can be prevented by targeting $120 \mathrm{~mm} \mathrm{Hg}$ instead of $140 \mathrm{~mm}$ $\mathrm{Hg}$ (corresponding to a number needed to treat of 125). Targeting $120 \mathrm{~mm} \mathrm{Hg}$ would result in higher incidence of CKD, AKI, hypotension, syncope and injurious falls. For example, in the same group of 10000 people, 118 additional cases of incident CKD would be caused by targeting $120 \mathrm{~mm} \mathrm{Hg}$ instead of $140 \mathrm{~mm} \mathrm{Hg}$ (corresponding to a number needed to harm of 85).

\section{Benefit-harm balance of blood pressure targets in subgroups with population weights}

We explored 120 subgroups defined by age (50 to 64,65 to 74,75 to 84 years), gender (male, female), prior history of diabetes (yes, no), CKD (no: estimated glomerular filtration rate $>60$, stage $3 \mathrm{~A}: 45$ to 59 , stage $3 \mathrm{~B}$ : 30 to 44 , stage 4: 15 to $29 \mathrm{~mL} / \mathrm{min} / 1.73 \mathrm{~m}^{2}$ ), chronic heart failure (yes, no) and stroke (yes, no). Furthermore, a web-calculator is available with which the impact of further risk factors and individual preferences can be explored (https://www. ebpi.uzh.ch/en/translational_research/archive_translational_research/archive_health_research_methods/ benefit_harm_assessments.html).

In table 3 , the probability that a lower SBP target of 120 or $<130 \mathrm{~mm} \mathrm{Hg}$ is better than a higher target of 140 or 131 to $149 \mathrm{~mm} \mathrm{Hg}$ is shown for all subgroups, considering all 
Table 2 Number of events in 10000 persons without prior history of stroke over 5 years

\begin{tabular}{|c|c|c|c|c|c|c|c|c|c|}
\hline \multirow[b]{2}{*}{ Target $(\mathrm{mm} \mathrm{Hg})$} & \multicolumn{3}{|c|}{ Age 50-64 } & \multicolumn{3}{|c|}{ Age $65-74$} & \multicolumn{3}{|c|}{ Age 75-84 } \\
\hline & 140 & 120 & Difference & 140 & 120 & Difference & 140 & 120 & Difference \\
\hline Death & 361 & 281 & +80 & 852 & 694 & +158 & 2037 & 1661 & +376 \\
\hline Myocardial infarction & 187 & 158 & +29 & 320 & 274 & +46 & 462 & 401 & +61 \\
\hline Heart failure & 195 & 131 & +64 & 567 & 402 & +165 & 763 & 543 & +220 \\
\hline Stroke & 104 & 91 & +13 & 246 & 208 & +38 & 443 & 385 & +58 \\
\hline ESRD & 50 & 32 & +18 & 68 & 43 & +25 & 60 & 39 & +21 \\
\hline CKD & 126 & 244 & -118 & 192 & 371 & -179 & 209 & 410 & -201 \\
\hline AKI & 194 & 332 & -138 & 189 & 325 & -136 & 177 & 308 & -131 \\
\hline Hypotension & 253 & 463 & -210 & 285 & 551 & -266 & 382 & 733 & -351 \\
\hline Syncope & 292 & 424 & -132 & 275 & 401 & -126 & 552 & 809 & -257 \\
\hline Injurious falls & 528 & 535 & -7 & 728 & 741 & -13 & 2022 & 2082 & -60 \\
\hline
\end{tabular}

Number of expected events in 10000 people without prior history of stroke over 5 years with a target of $140 \mathrm{~mm} \mathrm{Hg} \mathrm{or} 120 \mathrm{~mm} \mathrm{Hg}$, and the difference in numbers of events between the two targets.

AKI, acute kidney injury; CKD, chronic kidney disease; ESRD, end-stage renal disease.

available outcomes and accounting for statistical uncertainty of the estimates. In the main analysis, the probability that the lower target is better than the higher target was greater than 0.6 for 72 of 120 subgroups (blue cells: additional analyses with different assumptions for baseline incidences also yielded a probability above 0.6 , see online supplementary table S5). Notably, this was true for all male subgroups aged 75 to 84 without prior stroke, and for all subgroups of people with prior stroke aged 50 to 64 years.

The probability that $120 \mathrm{~mm} \mathrm{Hg}$ is better than $140 \mathrm{~mm}$ $\mathrm{Hg}$ was less than 0.4 for eight subgroups - these eight subgroups were women aged 50 to 74 who have a prior history of CKD stage 3B or 4 and without history of stroke (orange cells). The different results between subgroups partly arise due to lower cardiovascular risk in women and higher risk of AKI in people with CKD. In older people, the increased incidence of cardiovascular events and death drive the benefit-harm balance (data not shown). In subgroups with prior history of stroke, the probability that $<130 \mathrm{~mm} \mathrm{Hg}$ is better than 131 to $149 \mathrm{~mm} \mathrm{Hg}$ was never lower than 0.4 .

The mean index describes the clinical relevance of net benefit or harm as the mean number of events (with equivalent importance as death) that were prevented or caused by targeting $120 \mathrm{~mm} \mathrm{Hg}$ instead of $140 \mathrm{~mm} \mathrm{Hg}$ (see online supplementary table S6). Across subgroups, the maximum was 1133 with a possible range of 828 to 1193 (based on different assumptions for baseline risks) prevented events among 10000 people over 5 years, attained in the subgroup of men aged 75 to 84, without history of stroke, but with history of diabetes, heart failure and stage $3 \mathrm{~A}$ CKD.

\section{Sensitivity analyses considering individual preferences}

The impact of the variation in individual preferences (sensitivity analysis 1) is shown in figure 2. For eight subgroups the probability that the lower target is better was always greater than 0.6 - that is, for these subgroups, with every possible preference found in the survey, the better target was always $120 \mathrm{~mm} \mathrm{Hg}$. In many subgroups without prior history of stroke, especially with ages 50 to 64 years, the probability of $120 \mathrm{~mm} \mathrm{Hg}$ to be better than $140 \mathrm{~mm} \mathrm{Hg}$ almost spanned the full range of 0 to 1 , as indicated by the whiskers in figure 2. This meant that any probability of $120 \mathrm{~mm} \mathrm{Hg}$ being better or worse was possible, and depended on the preference of the individual. For example, using weights from a survey respondent who worried most about ESRD, followed by stroke and AKI, we calculated a probability of 0.05 that $120 \mathrm{~mm}$ $\mathrm{Hg}$ is better for a woman with diabetes, stage $4 \mathrm{CKD}$ and heart failure but no prior history of stroke. For the same subgroup, using weights from a survey respondent for whom myocardial infarction, heart failure and stroke were the most worrisome outcomes, the probability was 0.95 that $120 \mathrm{~mm} \mathrm{Hg}$ is better. For people with prior history of stroke, the range of the probability that $<130 \mathrm{~mm} \mathrm{Hg}$ is better than 131 to $149 \mathrm{~mm} \mathrm{Hg}$ was also wide. Thus, the probability that $<130 \mathrm{~mm} \mathrm{Hg}$ is better was dependent on the preferences of the individual. Sensitivity analyses 3 to 5 (see online supplementary tables $S 7-10$ ) confirmed the results of sensitivity analysis 1 . A sensitivity analysis with equal weights for all outcomes is shown in online supplementary table S11.

\section{Sensitivity analyses considering individual preferences and additionally including treatment burden}

In a further sensitivity analysis (sensitivity analysis 2), we added treatment burden, one of the patient-important outcomes for which we did not have data. Based on the preference survey, we assumed $20 \%$ of people would have more treatment burden with a lower blood pressure target due to needing an additional medication. While considering treatment burden decreased the median 
Table 3 Benefit-harm balance of different systolic blood pressures: probability that the lower target is better

\begin{tabular}{|c|c|c|c|c|c|}
\hline Diabetes & CKD & CHF & $\begin{array}{l}\text { Age } \\
50-64\end{array}$ & $\begin{array}{l}\text { Age } \\
65-74\end{array}$ & $\begin{array}{l}\text { Age } \\
75-84\end{array}$ \\
\hline
\end{tabular}

120 vs $140 \mathrm{~mm} \mathrm{Hg}$, SPRINT Probability that lower target is better in men without stroke \& without diabetes

$\begin{array}{llllll}\text { No } & \text { No } & \text { No } & 0.96 & 1.00 & 1.00 \\ \text { No } & \text { No } & \text { Yes } & 0.72 & 0.91 & 0.96 \\ \text { No } & \text { Stage 3A } & \text { No } & 0.96 & 1.00 & 1.00 \\ \text { No } & \text { Stage 3B } & \text { No } & 0.53 & 0.92 & 0.99 \\ \text { No } & \text { Stage 4 } & \text { No } & 0.48 & 0.80 & 0.97 \\ \text { No } & \text { Stage 3A } & \text { Yes } & 0.87 & 0.95 & 0.97 \\ \text { No } & \text { Stage 3B } & \text { Yes } & 0.43 & 0.74 & 0.89 \\ \text { No } & \text { Stage 4 } & \text { Yes } & 0.41 & 0.64 & 0.82\end{array}$

120 vs $140 \mathrm{~mm} \mathrm{Hg}$, SPRINT In women without stroke \& without diabetes

$\begin{array}{llllll}\text { No } & \text { No } & \text { No } & 0.63 & 0.98 & 1.00 \\ \text { No } & \text { No } & \text { Yes } & 0.27 & 0.75 & 0.94 \\ \text { No } & \text { Stage 3A } & \text { No } & 0.77 & 1.00 & 1.00 \\ \text { No } & \text { Stage 3B } & \text { No } & 0.12 & 0.69 & 0.99 \\ \text { No } & \text { Stage 4 } & \text { No } & 0.26 & 0.55 & 0.93 \\ \text { No } & \text { Stage 3A } & \text { Yes } & 0.57 & 0.92 & 0.97 \\ \text { No } & \text { Stage 3B } & \text { Yes } & 0.06 & 0.34 & 0.80 \\ \text { No } & \text { Stage 4 } & \text { Yes } & 0.20 & 0.31 & 0.60\end{array}$

120 vs $140 \mathrm{~mm} \mathrm{Hg}$, ACCORD In men without stroke \& with diabetes

$\begin{array}{llllll}\text { Yes } & \text { No } & \text { No } & 0.71 & 0.89 & 0.93 \\ \text { Yes } & \text { No } & \text { Yes } & 0.89 & 0.97 & 0.98 \\ \text { Yes } & \text { Stage 3A } & \text { No } & 0.92 & 0.95 & 0.96 \\ \text { Yes } & \text { Stage 3B } & \text { No } & 0.66 & 0.85 & 0.91 \\ \text { Yes } & \text { Stage 4 } & \text { No } & 0.45 & 0.74 & 0.89 \\ \text { Yes } & \text { Stage 3A } & \text { Yes } & 0.98 & 0.99 & 0.99 \\ \text { Yes } & \text { Stage 3B } & \text { Yes } & 0.88 & 0.97 & 0.97 \\ \text { Yes } & \text { Stage 4 } & \text { Yes } & 0.71 & 0.93 & 0.96\end{array}$

120 vs $140 \mathrm{~mm} \mathrm{Hg}$, ACCORD In women without stroke \& with diabetes

$\begin{array}{llllll}\text { Yes } & \text { No } & \text { No } & 0.37 & 0.70 & 0.81 \\ \text { Yes } & \text { No } & \text { Yes } & 0.69 & 0.89 & 0.92 \\ \text { Yes } & \text { Stage 3A } & \text { No } & 0.66 & 0.85 & 0.88 \\ \text { Yes } & \text { Stage 3B } & \text { No } & 0.31 & 0.54 & 0.73 \\ \text { Yes } & \text { Stage 4 } & \text { No } & 0.33 & 0.51 & 0.69 \\ \text { Yes } & \text { Stage 3A } & \text { Yes } & 0.92 & 0.97 & 0.97 \\ \text { Yes } & \text { Stage 3B } & \text { Yes } & 0.63 & 0.84 & 0.91 \\ \text { Yes } & \text { Stage 4 } & \text { Yes } & 0.57 & 0.78 & 0.88\end{array}$

$<130$ vs $131-149 \mathrm{~mm} \mathrm{Hg}, \quad$ In men with stroke SPS3

\begin{tabular}{llllll} 
No & No & No & 0.70 & 0.58 & 0.44 \\
No & No & Yes & 0.68 & 0.60 & 0.49 \\
Yes & No & No & 0.68 & 0.61 & 0.48 \\
Yes & No & Yes & 0.66 & 0.59 & 0.54 \\
\hline
\end{tabular}

Continued
Table 3 Continued

\begin{tabular}{|c|c|c|c|c|c|}
\hline Diabetes & CKD & CHF & $\begin{array}{l}\text { Age } \\
50-64\end{array}$ & $\begin{array}{l}\text { Age } \\
65-74\end{array}$ & $\begin{array}{l}\text { Age } \\
75-84\end{array}$ \\
\hline \multicolumn{3}{|c|}{$\begin{array}{l}<130 \text { vs } 131-149 \mathrm{~mm} \mathrm{Hg} \text {, } \\
\text { SPS3 }\end{array}$} & \multicolumn{3}{|c|}{ In women with stroke } \\
\hline No & No & No & 0.71 & 0.59 & 0.40 \\
\hline No & No & Yes & 0.69 & 0.60 & 0.46 \\
\hline Yes & No & No & 0.69 & 0.60 & 0.46 \\
\hline Yes & No & Yes & 0.67 & 0.62 & 0.50 \\
\hline
\end{tabular}

The probability that the lower target $(120 \mathrm{~mm} \mathrm{Hg}$ or $<130 \mathrm{~mm} \mathrm{Hg})$ is a better target than the higher target $(140 \mathrm{~mm} \mathrm{Hg}$ or 131 to $149 \mathrm{~mm}$ $\mathrm{Hg}$ ) is shown for all subgroups (calculated from 100000 repetitions). Subgroups are presented according to which RCT was used to inform the analysis and what targets were compared. A probability of 0.5 means that both targets have the same benefit-harm balance. Blue colour indicates the lower target was better also in additional analysis with different assumptions for baseline incidences, and orange colour indicates the higher target was better also in the additional analyses. We did not calculate the benefit-harm balance of different blood pressure targets for people with prior history of stroke and CKD, because renal outcomes that would be important for people with CKD, but rare for people without CKD, were not reported by SPS3. $\mathrm{CHF}$, chronic heart failure; CKD, chronic kidney disease; RCT, randomised clinical trial.

probability that the lower target is better, it also widened the range of the probability in most subgroups (see online supplementary figure $\mathrm{S} 4$ ).

\section{DISCUSSION}

Our analyses showed that the benefit-harm balance of SBP targets in people with MCC and hypertension depends on age, gender, comorbidities (history of diabetes, CKD, heart failure and stroke) and largely on the outcome preferences of patients. Our results highlight the importance of performing qualitative and quantitative preference surveys to identify patient-important outcomes and determine weights, and to assess the impact of different preferences on the benefit-harm balance. Preferences should be assessed in the target population, and it is known that physicians can have different preferences. ${ }^{46}$ If there is large variation in individual preferences, there are two possible consequences. First, it is possible that one intervention is still consistently better, as was true for a few of the subgroups in our analyses. Second, the balance can be preference-sensitive, as was true for most of the subgroups in our analyses, where the probability of one target being better than the other spanned almost the full range of 0 to 1 , depending on the individual's preferences. In this case, shared decision-making with explicit determination of the individual's preferences may be appropriate. Further research is needed on how to elicit individual preferences in the clinic. Patient decision aids with an integrated calculation of the benefit-harm balance may help in shared decision-making when the benefit-harm balance is preference-sensitive and depends on prior medical history. The web-calculator that we provided as an addendum is not ready to be used as a decision aid 
A) People without prior history of stroke and diabetes (SPRINT)

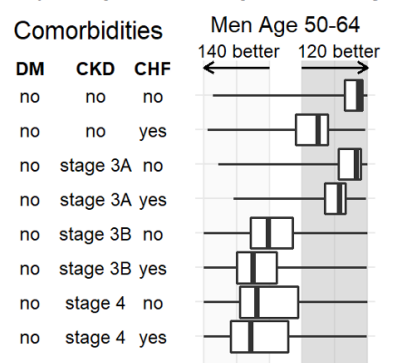

0.00 .20 .40 .60 .81 .0

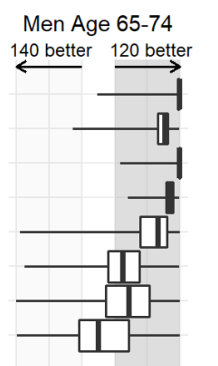

0.00 .20 .40 .60 .81 .0
Men Age 75-84

40 better 120 better
Women Age 50-64

140 better 120 bette

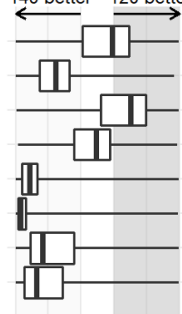

0.00 .20 .40 .60 .81 .0

Probability that $120 \mathrm{mmHg}$ is better than $140 \mathrm{mmHg}$

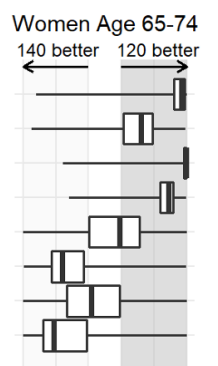

0.00 .20 .40 .60 .81 .0
Women Age 75-84 140 better 120 better
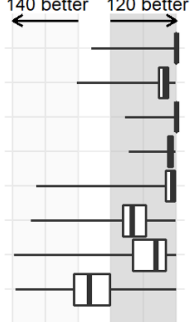

0.00 .20 .40 .60 .81 .0

B) People with diabetes but without prior history of stroke (ACCORD)

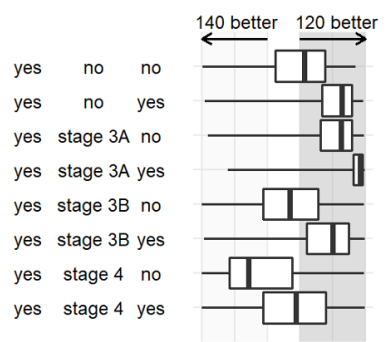

0.00 .20 .40 .60 .81 .0

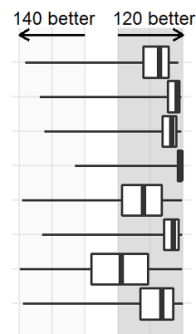

0.00 .20 .40 .60 .81 .0
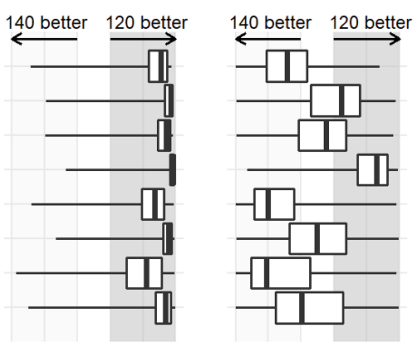

0.00 .20 .40 .60 .81 .0

Probability that $120 \mathrm{mmHg}$ is better than $140 \mathrm{mmHg}$
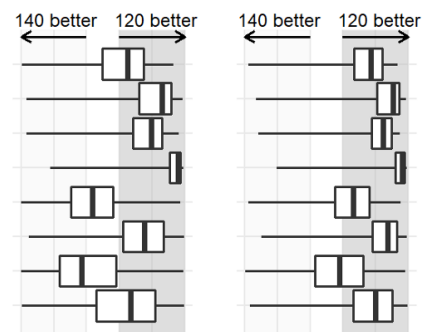

0.00 .20 .40 .60 .81 .0

0.00 .20 .40 .60 .81 .0

C) People with prior history of stroke (SPS3)

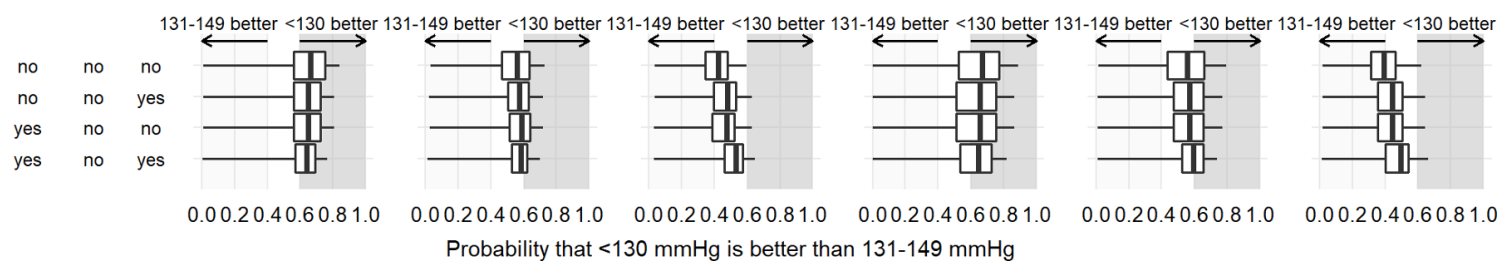

Figure 2 Sensitivity analysis 1 - impact of variation in preferences between individuals. Distribution (full range, IQR and median) of the benefit-harm balance with 207 different sets of weights, according to the preferences of all 207 individuals in the preference survey. In almost all subgroups, the variation in individual preferences lead to wide ranges of balances, with some favouring a lower target (dark grey shaded), and some favouring a higher target (light grey shade). In eight subgroups without prior history stroke, even with the most extreme preferences, the better target was always $120 \mathrm{~mm} \mathrm{Hg}$. CHF, Chronic heart failure; CKD, Chronic kidney disease; DM, Diabetes mellitus type 2; IQR, interquartile range.

- for this, the tool would need to be extensively tested with patients and clinicians. It would also need to convey the uncertainty in the benefit-harm balance, for example showing estimates of the benefit-harm balance using different prediction models for baseline incidences.

While for most subgroups the benefit-harm balance of SBP targets was preference-sensitive, and shared decision-making would accordingly be appropriate, for a few subgroups, one target was clearly better. We identified eight subgroups without prior history of stroke for which a target of $120 \mathrm{~mm} \mathrm{Hg}$ was better even for extreme preferences. The benefit-harm balance shifted only when we assumed that individuals perceived the additional treatment burden as high. The absolute benefit was relatively high, with 436 to 1133 events of equivalent importance to death prevented in 10000 people over 5 years.

Two guidelines about blood pressure targets have been published in 2017 from US organisations: the American College of Cardiology/American Heart Association (ACC/AHA) ${ }^{1}$ and the American College of Physicians (ACP). ${ }^{2}$ Neither performed a quantitative benefit-harm assessment with explicit weights. Their recommendations differ, both from each other and from our results, mostly due to four reasons. First, the ACC/AHA guideline did not consider any adverse events except the combined outcome 'renal outcomes' (including ESRD and AKI) that is, the guideline made a trade-off between cardiovascular benefit and renal harm, but did not take into account other harms of lower targets like syncope. Second, the ACC/AHA guideline considered only relative risk reductions in the underlying systematic review, and it is unclear how it considered absolute effects of different targets. Third, both guidelines assessed achieved blood pressure additionally, which we did not consider, because these values can deviate from the target for a large number of reasons, and were not randomised in the trials. Fourth, the implicit weights that were assigned differed, as we interpret from the published rationale. The ACC/AHA guideline implicitly weighs cardiovascular events more than possible adverse events - which may reflect the preferences of many, but not all patients. The ACP guideline implicitly weighs treatment burden and adverse events 
more than the ACC/AHA guideline, and states that while $<140 \mathrm{~mm} \mathrm{Hg}$ would further reduce the cardiovascular risk in people aged 60 or older, the additional benefit is small and inconsistent across outcomes.

Notwithstanding our different methods and results, we agree on many points with both guidelines. Our results indicate that indeed individual assessment of benefits and harms in people with MCC is important, like the ACP guideline recommends, and individual preferences should be considered. The ACC/AHA guideline sees significant benefit of a lower SBP target, but adjusts the target of $120 \mathrm{~mm} \mathrm{Hg}$ to $130 \mathrm{~mm} \mathrm{Hg}$ because in real-world practice, achieved blood pressure measurements tend to be higher than in the setting of a RCT. ${ }^{10}$ Similarly, our results indicate that a SBP target of $120 \mathrm{~mm} \mathrm{Hg}$ has significant potential to benefit subgroups without prior history of stroke and no CKD of stage $3 \mathrm{~B}$ or higher. In contrast, a SBP target of $140 \mathrm{~mm} \mathrm{Hg}$ was better for some subgroups of women aged 50 to 64 with CKD stages $3 \mathrm{~B}$ or 4 . The ACC/AHA guideline recommends a target of $130 \mathrm{~mm} \mathrm{Hg}$ for people with prior history of CKD, which may reflect that most cases of CKD are of stage $3 \mathrm{~A},{ }^{44}$ where lower targets may be more beneficial.

Our approach to identify the optimal SBP target for people with MCC had several strengths. First, we predefined the subgroups of relevance for people with MCC. Second, in collaboration with patients and caregivers, we identified patient-important outcomes, and regularly informed them about the progress to assure the results would be meaningful to people with MCC. Third, we performed sensitivity analyses with different weights and could show in which subgroups the benefit-harm balance could shift, and in which the balance was relatively stable. Finally, to quantify the uncertainty in the probability that one target is better than another, we performed additional analyses including all assumptions that we were unsure about, to get a conservative estimate of what the range of possible estimates may be.

Our analyses emphasise where most uncertainty remains. Evidence is lacking for cognitive impairment and for mild adverse events, such as daily mild dizziness. SPS3 did not report heart failure, ESRD and AKI, which adds uncertainty for people with prior history of stroke. As these outcomes are particularly important in people with CKD, the benefit-harm balance for people with prior history of stroke and CKD cannot be judged. People with heart failure were not well represented in the trials, therefore, uncertainty is larger for subgroups with heart failure. Furthermore, our main analysis did not include treatment burden, as we would have needed to know how many more people experience significantly more treatment burden with lower targets. However, our analyses indicate treatment burden may not shift the benefit-harm balance drastically on average, but it may increase the impact of variation in individual preferences.

Our model puts the most applicable, valid and precise evidence into context. To address this gap in evidence in a study instead, a very large RCT would be needed, in which older people with MCCs - and in different combinations of MCCs - are well represented, and which includes a preference survey among participants. Chances are no such RCT will ever be performed. A major limitation is, therefore, that it is difficult to confirm the results of the model. However, in the absence of a study, a model that follows a systematic approach based on epidemiological principles and the available clinical evidence is necessary to inform this question. We aimed to assess the benefit-harm balance of different blood pressure targets; how these targets are best achieved was out of scope. Techniques to measure the blood pressure differed between trials and may differ between trials and clinical practice. ${ }^{10}$ Therefore, we presented the results using different trials separately, because their targets may be interpreted differently. As the target setting was the USA and we used baseline risks applicable there, the benefit-harm balance of SBP targets may be different in other countries.

\section{CONCLUSIONS}

In conclusion, the lower SBP targets have significant potential to benefit people with MCC who tolerate antihypertensive treatment, especially among those who do not have CKD stage $3 \mathrm{~B}$ or higher and no history of ischaemic stroke. The potential benefit is largest in people older than 75 who do not have a history of ischaemic stroke. Shared decision-making should take place, as individual preferences can have a substantial influence on the benefit-harm balance.

\section{Author affiliations}

${ }^{1}$ Epidemiology, Biostatistics and Prevention Institute, University of Zurich, Zurich, Switzerland

${ }^{2}$ Division of Geriatric Medicine and Gerontology, Center for Transformative Geriatric Research, Johns Hopkins University School of Medicine, Baltimore, Maryland, USA ${ }^{3}$ Center for Clinical Information Services, Kaiser Permanente Care Management Institute, Oakland, California, USA

${ }^{4}$ Kaiser Permanente National Guideline Program, Oakland, California, USA ${ }^{5}$ Guidelines International Network, Board of Trustees, Denver, Colorado, USA

${ }^{6}$ Family Medicine, Colorado Permanente Medical Group, Denver, Colorado, USA

${ }^{7}$ Clinical Education MOC Portfolio, The Permanente Federation, Oakland, California, USA

${ }^{8}$ The Center for Health Research, Kaiser Permanente Northwest, Northwest Permanente Research and Evaluation, Portland, Oregon, USA

${ }^{9}$ Department of Pulmonary \& Critical Care Medicine, Northwest Permanente, Portland, Oregon, USA

${ }^{10}$ Oregon Health \& Science University, Portland, Oregon, USA

${ }^{11}$ Kaiser Permanente Northwest, National Guideline Program, Portland, Oregon, USA

${ }^{12}$ Division of Geriatric Medicine and Gerontology, Center on Aging and Health, Johns Hopkins University School of Medicine, Baltimore, Maryland, USA

${ }^{13}$ Department of Health Policy and Management, Johns Hopkins Bloomberg School of Public Health, Baltimore, Maryland, USA

${ }^{14}$ Division of General Internal Medicine, Johns Hopkins University School of Medicine, Baltimore, Maryland, USA

${ }^{15}$ Institute for Health Research, Kaiser Permanente, Denver, Colorado, USA

${ }^{16}$ Department of Family Medicine, University of Colorado School of Medicine, Aurora, Colorado, USA

${ }^{17}$ Department of Public Health College of Medicine, National Cheng Kung University, Tainan, Taiwan

${ }^{18}$ Division of Geriatric Medicine and Gerontology, Patient and Caregiver Partner Group, Johns Hopkins University School of Medicine, Baltimore, Maryland, USA 
${ }^{19}$ Gerontological Society of America, Washington, District of Columbia, USA

${ }^{20}$ Family Caregiver Advocacy, Kensington, Maryland, USA

Acknowledgements This article was prepared using the SPRINT_POP Research Materials obtained from the NHLBI Biologic Specimen and Data Repository Information Coordinating Center and does not necessarily reflect the opinions or views of the SPRINT_POP or the NHLBI.

Contributors CMB, CWR, WVC, WLB, KA, CG, KM, SM and MAP acquired the funding for the project. HEA and RFW curated the data, HEA analysed the data and MAP contributed to the data analysis. HEA, CMB, OCS, RFW and TY contributed to literature searches and data extraction. HEA, CMB, CWR, RAM, WVC, OCS, RFW, WLB, EAB, TY, BL and MAP contributed to the development of the model. CMB and RFW performed project administration. CMB, RFW and MAP supervised the project. HEA, CMB and MAP drafted the original manuscript. All authors contributed to the conceptualisation of the study, reviewed and edited the manuscript and approved the final manuscript.

Funding Research reported in this article was funded through a Patient-Centered Outcomes Research Institute (PCORI) Award (ME-1310-07619). The statements in this article are solely the responsibility of the authors and do not necessarily represent the views of the Patient-Centered Outcomes Research Institute (PCORI), its Board of Governors or Methodology Committee. Hélène Aschmann was also supported through a PhD fellowship of the Béatrice Ederer-Weber Foundation.

Competing interests None declared.

Patient consent for publication Not required.

Ethics approval This modelling study is based on published data except for the patient preference survey. Both the Institutional Review Boards of Johns Hopkins University (00114695) and Kaiser Permanente Colorado (C0-15-2195) approved the survey.

Provenance and peer review Not commissioned; externally peer reviewed.

Data availability statement Data are available upon reasonable request.

Open access This is an open access article distributed in accordance with the Creative Commons Attribution Non Commercial (CC BY-NC 4.0) license, which permits others to distribute, remix, adapt, build upon this work non-commercially, and license their derivative works on different terms, provided the original work is properly cited, appropriate credit is given, any changes made indicated, and the use is non-commercial. See: http://creativecommons.org/licenses/by-nc/4.0/.

\section{REFERENCES}

1. Whelton PK, Carey RM, Aronow WS, et al. 2017 ACC/AHA/AAPAV ABC/ACPM/AGS/APhA/ASH/ASPC/NMA/PCNA Guideline for the prevention, detection, evaluation, and management of high blood pressure in adults: executive summary. J Am Coll Cardiol 2018;71:2199-269.

2. Qaseem A, Wilt TJ, Rich R, et al. Pharmacologic treatment of hypertension in adults aged 60 years or older to higher versus lower blood pressure targets: a clinical practice guideline from the American College of physicians and the American Academy of family physicians. Ann Intern Med 2017;166:430-7.

3. James PA, Oparil S, Carter BL, et al. 2014 Evidence-based guideline for the management of high blood pressure in adults, report from the panel members appointed to the eighth joint National Committee (JNC8). JAMA 2013;1097:1-14.

4. Mancia G, Fagard R, Narkiewicz K, et al. 2013 ESH/ESC guidelines for the management of arterial hypertension: the task force for the management of arterial hypertension of the European Society of hypertension (ESH) and of the European Society of cardiology (ESC). Eur Heart J 2013;34:2159-219.

5. Cushman WC, Evans GW, Byington RP, et al. Effects of intensive blood-pressure control in type 2 diabetes mellitus. N Engl $J$ Med 2010;362:1575-85.

6. Wright JT, Williamson JD, Whelton PK, et al. A randomized trial of intensive versus standard blood-pressure control. N Engl J Med 2015;373:2103-16.

7. The SPS3 Study Group. Blood-Pressure targets in patients with recent lacunar stroke: the SPS3 randomised trial. Lancet 2013;382:507-15.

8. Peralta CA, McClure LA, Scherzer R, et al. Effect of intensive versus usual blood pressure control on kidney function among individuals with prior lacunar stroke: a post hoc analysis of the secondary prevention of small subcortical strokes (SPS3) randomized trial. Circulation 2016;133:584-91.

9. Ortiz E, James PA. Let's not sprint to judgment about new blood pressure goals. Ann Intern Med 2016;164:692.

10. Weiss J, Freeman M, Low A, et al. Benefits and harms of intensive blood pressure treatment in adults aged 60 years or older: a systematic review and meta-analysis. Ann Intern Med 2017; 166:419-29.

11. Bennett WL, Robbins CW, Bayliss EA, et al. Engaging stakeholders to inform clinical practice guidelines that address multiple chronic conditions. J Gen Intern Med 2017;32:883-90.

12. Fain $\mathrm{KM}, \mathrm{Yu} \mathrm{T}, \mathrm{Li} \mathrm{T}$, et al. Evidence selection for a prescription drug's benefit-harm assessment: challenges and recommendations. J Clin Epidemiol 2016;74:151-7.

13. Gail MH, Costantino JP, Bryant J, et al. Weighing the risks and benefits of tamoxifen treatment for preventing breast cancer. $J$ Natl Cancer Inst 1999;91:1829-46.

14. Puhan MA, Singh S, Weiss CO, et al. A framework for organizing and selecting quantitative approaches for benefit-harm assessment. BMC Med Res Methodol 2012;12:173.

15. Husereau D, Drummond M, Petrou S, et al. Consolidated Health Economic Evaluation Reporting Standards (CHEERS) - explanation and elaboration: a report of the ISPOR health economic evaluation publication guidelines good reporting practices task force. Value Health 2013;16:231-50.

16. Stegeman I, Bossuyt PM, Yu T, et al. Aspirin for primary prevention of cardiovascular disease and cancer. A benefit and harm analysis. PLoS One 2015;10:e0127194-12.

17. Puhan MA, Yu T, Stegeman I, et al. Benefit-harm analysis and charts for individualized and preference-sensitive prevention: example of low dose aspirin for primary prevention of cardiovascular disease and cancer. BMC Med 2015;13:250.

18. Yu T, Fain K, Boyd CM, et al. Benefits and harms of roflumilast in moderate to severe COPD. Thorax 2014;69:616-22.

19. Yebyo HG, Aschmann HE, Puhan MA. Finding the balance between benefits and harms when using statins for primary prevention of cardiovascular disease: a modeling study. Ann Intern Med 2018. doi: 10.7326/M18-1279. [Epub ahead of print 04 Dec 2018].10.7326/ M18-1279.

20. Hannson L. The BBB Study: the effect of intensified antihypertensive treatment on the level of blood pressure, side-effects, morbidity and mortality in "well-treated" hypertensive patients. Behandla Blodtryck Bättre. Blood Press 1994;3:248-54.

21. Sarnak MJ, Greene T, Wang X, et al. The effect of a lower target blood pressure on the progression of kidney disease: long-term follow-up of the modification of diet in renal disease study. Ann Intern Med 2005;142:342-51.

22. Toto RD, Mitchell HC, Smith RD, et al. "Strict" blood pressure control and progression of renal disease in hypertensive nephrosclerosis. Kidney Int 1995;48:851-9.

23. Higgins JPT, Altman DG, Gøtzsche PC, et al. The Cochrane collaboration's tool for assessing risk of bias in randomised trials. BMJ 2011;343:d5928.

24. Estacio R, Coll J, Tran Z, et al. Effect of intensive blood pressure control with valsartan on urinary albumin excretion in normotensive patients with type 2 diabetes. Am J Hypertens 2006;19:1241-8.

25. Verdecchia P, Staessen JA, Angeli F, et al. Usual versus tight control of systolic blood pressure in non-diabetic patients with hypertension (Cardio-Sis): an open-label randomised trial. Lancet 2009;374:525-33.

26. Estacio RO, Jeffers BW, Hiatt WR, et al. The effect of nisoldipine as compared with enalapril on cardiovascular outcomes in patients with non-insulin-dependent diabetes and hypertension. N Engl J Med 1998;338:645-52.

27. Schrier RW, Estacio RO, Esler A, et al. Effects of aggressive blood pressure control in normotensive type 2 diabetic patients on albuminuria, retinopathy and strokes. Kidney Int 2002;61:1086-97.

28. Wright JT, Bakris G, Greene T, et al. Effect of blood pressure lowering and antihypertensive drug class on progression of hypertensive kidney disease: results from the AASK trial. JAMA 2002;288:2421-31.

29. Hansson L, Zanchetti A, Carruthers SG, et al. Effects of intensive blood-pressure lowering and low-dose aspirin in patients with hypertension: principal results of the hypertension optimal treatment (HOT) randomised trial. The Lancet 1998;351:1755-62.

30. Asayama K, Ohkubo T, Metoki H, et al. Cardiovascular outcomes in the first trial of antihypertensive therapy guided by self-measured home blood pressure. Hypertens Res 2012;35:1102-10.

31. Ruggenenti P, Perna A, Loriga G, et al. Blood-pressure control for renoprotection in patients with non-diabetic chronic renal disease (REIN-2): multicentre, randomised controlled trial. Lancet 2005;365:939-46. 
32. JATOS Study Group. Principal results of the Japanese trial to assess optimal systolic blood pressure in elderly hypertensive patients (JATOS). Hypertens Res 2008;31:2115-27.

33. Ogihara T, Saruta T, Rakugi $\mathrm{H}$, et al. Target blood pressure for treatment of isolated systolic hypertension in the elderly: valsartan in elderly isolated systolic hypertension study. Hypertension 2010;56:196-202.

34. Mant J, McManus RJ, Roalfe A, et al. Different systolic blood pressure targets for people with history of stroke or transient ischaemic attack: PAST-BP (Prevention After Stroke-Blood Pressure) randomised controlled trial. BMJ 2016;352352.

35. Wei Y, Jin Z, Shen G, et al. Effects of intensive antihypertensive treatment on Chinese hypertensive patients older than 70 years. $J$ Clin Hypertens 2013;15:420-7.

36. Schrier R, McFann K, Johnson A, et al. Cardiac and renal effects of standard versus rigorous blood pressure control in autosomaldominant polycystic kidney disease: results of a seven-year prospective randomized study. J Am Soc Nephrol 2002;13:1733-9.

37. UK Prospective Diabetes Study Group. Tight blood pressure control and risk of macrovascular and microvascular complications in type 2 diabetes: UKPDS 38. UK prospective diabetes Study Group. BMJ 1998;317:703-13.

38. Greenland S, Mansournia MA, Altman DG. Sparse data bias: a problem hiding in plain sight. BMJ 2016;22.
39. Greenland S. Bayesian perspectives for epidemiological research: I. Foundations and basic methods. Int J Epidemiol 2006;35:765-75.

40. National institutes of health National Heart Lung and Blood Institute. Incidence \& Prevalence: 2006 Chart Book on Cardiovascular and Lung Diseases. Available: http://www.bls.gov/oes/2010/may/stem. htm [Accessed 18 May 2017].

41. Agarwal SK, Chambless LE, Ballantyne CM, et al. Prediction of incident heart failure in general practice: the Atherosclerosis risk in communities (ARIC) study. Circ Heart Fail 2012;5:422-9.

42. Chambless LE, Heiss G, Shahar E, et al. Prediction of ischemic stroke risk in the Atherosclerosis risk in Communities study. Am J Epidemiol 2004;160:259-69.

43. Kshirsagar AV, Bang $\mathrm{H}$, Bomback AS, et al. A simple algorithm to predict incident kidney disease. Arch Intern Med 2008;168:2466-73.

44. Grams ME, Astor BC, Bash LD, et al. Albuminuria and estimated glomerular filtration rate independently associate with acute kidney injury. JASN 2010;21:1757-64.

45. Yu T, Holbrook JT, Thorne JE, et al. Outcome preferences in patients with noninfectious uveitis: results of a Best-Worst scaling study. Invest Ophthalmol Vis Sci 2015:56:6864-72.

46. McAlister FA, O'Connor AM, Wells G, et al. When should hypertension be treated? The different perspectives of Canadian family physicians and patients. CMAJ 2000;163:403-8. 